\title{
Aplicações da cirurgia videolaparoscópica em crianças
}

\author{
Applications of videolaparoscopic surgery in children \\ Edward Esteves ${ }^{1}$, Miguel O. Neto ${ }^{2}$, Eriberto C. Neto ${ }^{2}$, Osmar Terêncio Jr. ${ }^{3}$, \\ Bernardina B. Carvalho ${ }^{4}$, Ruy E. Pereira ${ }^{5}$
}

\section{Resumen}

Objetivo: apresentar a casuística de videolaparoscopias do serviço, analisando as principais indicações para operações videolaparoscópicas em pediatria e considerando as vantagens e desvantagens em relação aos procedimentos convencionais por laparotomia.

Métodos: análise retrospectiva de 612 videolaparoscopias em crianças com idades de 8 dias a 17 anos, operadas de novembro/1995 a 2000. São descritos os princípios básicos da videolaparoscopia e o manejo pós-operatório nas inúmeras indicações pediátricas. Os resultados, vantagens e complicações foram analisados após um período de seguimento de até 5 anos.

Resultados: a cirurgia laparoscópica permitiu realizar vários procedimentos abdominais convencionalmente efetuados por via aberta, sobretudo no tratamento do refluxo gastroesofágico, abdome agudo inflamatório, criptorquidia, obstrução por bridas, litíase biliar, tumores, afecções de ovário, esplenectomia, aganglionose, trauma e outros. A morbidade foi baixa (1\%) e a mortalidade devido à laparoscopia foi nula. Houve conversão para laparotomia em somente 14 casos $(2,3 \%)$, sobretudo em trauma. As principais vantagens incluíram mínimos trauma cirúrgico, dor e íleo paralítico, pouco tempo de internação, quase ausência de hérnia incisional e melhor estética.

Conclusões: a videolaparoscopia parece ser um grande avanço da cirurgia pediátrica moderna, permitindo tratar de modo menos invasivo e com segurança grande parte das afecções abdominais em crianças de todas as idades.

J Pediatr (Rio J) 2001; 77 (5): 407-12: cirurgia pediátrica, videolaparoscopia, refluxo gastroesofágico, abdome agudo, trauma.

\section{Introdução}

Os avanços da cirurgia pediátrica representam sempre considerável melhora no tratamento das crianças em geral. Até recentemente todas as afecções intra-abdominais de tratamento cirúrgico necessitavam de laparotomia, com a

\footnotetext{
1. Professor de Cirurgia Pediátrica da Universidade Federal de Goiás (UFG) e Mestre em Cirurgia pela Universidade Federal de São Paulo.

2. Cirurgião pediátrico no Serviço de Cirurgia Pediátrica da UFG.

3. Cirurgião gastroenterológico no Serviço de Trauma do Instituto Ortopédico de Goiânia e no Hospital Amparo.

4. Residente de cirurgia pediátrica da UFG.

5. Professor e Chefe do Serviço de Cirurgia Pediátrica da UFG.
}

Abstract
Objectives: to present the videolaparoscopies performed by the Children's Surgery Service, and study the main indications for pediatric laparoscopic surgeries, considering advantages and disadvantages over conventional open procedures.

Methods: retrospective analysis of 612 videolaparoscopies in children aged between 8 days and 17 years treated from November/ 95 to 2000 . Basic principles of videolaparoscopy and the postoperative management of several pediatric diseases are described. The results, advantages, and complications were analyzed after a 5-year follow-up period.

Results: laparoscopic surgery allowed for a wide series of abdominal procedures conventionally carried out through open surgery, mainly for the treatment of gastroesophageal reflux disease, inflammatory acute abdomen, adhesive intestinal obstruction, biliary lithiasis, tumors, cryptorchidism, ovarian diseases, splenectomies, aganglionosis, trauma and others. Morbidity was low (1\%), and mortality due to laparoscopy was nonexistent. Conversion to laparotomy occurred in only 14 cases $(2.3 \%)$, mainly because of trauma. The principal advantages included minimal surgical trauma, pain and reflex ileum, short hospital stay, almost no incisional hernias and better cosmetic scars.

Conclusions: videolaparoscopy seems to be a great advance in modern pediatric surgery, allowing safer and less invasive treatment of a wide series of abdominal diseases at all pediatric ages.

J Pediatr (Rio J) 2001; 77 (5): 407-12: pediatric surgery, videolaparoscopy, gastroesophageal reflux, acute abdomen, trauma.

secção de todas camadas da parede abdominal e exposição das vísceras, com diversas possibilidades de complicação e inconvenientes pós-operatórios, como hemorragia, internação prolongada, infecção e cicatrizes indesejáveis. A laparoscopia era aplicada apenas como método diagnóstico. Com o advento e as inúmeras vantagens da cirurgia minimamente invasiva, no fim da década de 80 , em adultos, utilizando-se microcâmera acoplada aos laparoscópios, monitor de vídeo e instrumental fino sem abertura do abdome, a miniaturização dos equipamentos permitiu a aplicação em escala progressiva da videocirurgia em crianças ${ }^{1}$. 
Os autores apresentam os princípios básicos do tratamento e sua experiência em videolaparoscopia pediátrica com instrumental especialmente confeccionado para uso em crianças, analisando as principais indicações e as vantagens sobre as operações com cavidade aberta.

\section{Métodos}

No período de novembro/95 a novembro/2000, 588 crianças com idades de 8 dias a 17 anos, sendo 332 do sexo feminino e 256 do masculino, foram submetidas a 612 laparoscopias por diversas afecções, resumidas na Tabela 1.

Tabela 1 - Indicações de cirurgia laparoscópica pediátrica

\begin{tabular}{|c|c|}
\hline Refluxo gastroesofágico patológico & 126 \\
\hline Apendicite aguda & 49 \\
\hline Diagnóstico em abdome agudo inflamatório & 45 \\
\hline \multirow{2}{*}{$\begin{aligned} \text { Criptorquia abdominal } & -1 \text { ou } 2 \text { tempos } \\
& - \text { Anorquia }\end{aligned}$} & 39 \\
\hline & 18 \\
\hline Biópsia / exérese de tumores & 40 \\
\hline Trauma abdominal fechado e aberto & 33 \\
\hline Colecistectomia & 24 \\
\hline Inspeção de hérnia contralateral & 21 \\
\hline Estadiamento de doença de Hodgkin & 16 \\
\hline Cistos ou tumores de ovário & 14 \\
\hline Obstrução por bridas & 14 \\
\hline Esplenectomia & 13 \\
\hline Investigação de icterícia neonatal & 12 \\
\hline Cirurgia de Malone (apendicostomia) & 9 \\
\hline Varicocele & 9 \\
\hline Dor abdominal recorrente & 9 \\
\hline Cistos de omento, mesentério, pseudocistos & 8 \\
\hline Genitália ambígua & 8 \\
\hline Anemia hemolítica: esplenect + colecistec. & 8 \\
\hline Complicação de cateter de diálise & 7 \\
\hline Complicação de cateter de DVP & 6 \\
\hline Intussuscepção & 6 \\
\hline Remoção de corpo estranho - vários & 6 \\
\hline Biópsia hepática/esplênica visão direta & 6 \\
\hline Má rotação intestinal & 5 \\
\hline Vagotomia (úlcera crônica em adolescente) & 5 \\
\hline Gastrostomia / ileostomia alimentar & 4 \\
\hline Aganglionose - colectomia & 4 \\
\hline - biópsias seriadas & 4 \\
\hline Hérnia diafragmática (Morgagni / parahiatal) & 4 \\
\hline Debridamento em pancreatite & 4 \\
\hline Duplicidades & 4 \\
\hline Nefrectomia & 4 \\
\hline Retopexia em prolapso de reto ou neovagina & 3 \\
\hline Volvo gástrico & 3 \\
\hline Atresia duodenal & 3 \\
\hline Divertículo de Meckel & 3 \\
\hline Pexia em prolapso de reto ou neovagina & 3 \\
\hline Outros $*$ & $13^{*}$ \\
\hline
\end{tabular}

* Hepatectomia parcial (2), estenose hipertrófica do piloro (2), tuberculose intestinal (2), cisto esplênico(2), hemangioma hepático (1), torção de epíploon (1), linfangioma mesentérico (1), acalasia de esôfago (1), baço errante (1).
O acesso laparoscópico era escolhido em casos em que habitualmente se indicaria laparotomia, dependendo de fatores como previsão de incisões da laparoscopia menores ou mais estéticas, crianças obesas, adolescentes, dúvida diagnóstica, biópsias, disponibilidade do aparelho ou acessórios, indicações laparoscópicas consagradas como colecistectomia, refluxo gastroesofágico e outros ${ }^{1,2}$. Casos de baixa morbidade operatória, rápida recuperação e bom aspecto estético por via aberta como hérnia inguinal e apendicectomia em meninos magros, assim como pacientes hemodinamicamente instáveis ou operações de múltiplas suturas (ex: ampliações vesicais, atresia de vias biliares) não foram selecionados para videolaparoscopia.

Técnica laparoscópica básica: estando a criança sob anestesia geral, realiza-se pequena incisão intra-umbilical para colocação de um trocarte sob visão direta do peritônio, ou introduz-se uma agulha de Veress na cicatriz umbilical. Pelo trocarte ou pela agulha é infundido $\mathrm{CO}_{2}$ na cavidade peritoneal com um insuflador automático. $\mathrm{O}$ pneumoperitônio expande-se até a pressão máxima de 8 a $10 \mathrm{mmHg}$, fixada previamente no insuflador, afasta a parede abdominal das vísceras e forma o espaço de trabalho na cavidade. $\mathrm{OCO}_{2}$ não é inflamável com eletrocautério e é rapidamente difusível para o ar expirado pelos pulmões. Durante toda a operação, a criança é monitorizada com oxi-capnógrafo.

Um trocarte de 4 ou $10 \mathrm{~mm}$ é introduzido no umbigo ou em outro ponto de melhor visão da área a ser operada. Por este trocarte, uma óptica conectada a uma microcâmera e uma fonte de luz permitem iluminare visibilizar a cavidade, mostrada em uma televisão ou monitor de vídeo. Sob visão interna da câmera, outros trocartes finos são passados pela parede por perfurações de $3 \mathrm{a} 5 \mathrm{~mm}$ conforme a operação proposta. A óptica aumenta a visão até 16 ou 20 vezes, ampliando-se detalhes mínimos no intra-operatório. Quase todas as operações foram gravadas em fitas de vídeo ou impressas em fotos.

Nos orifícios são introduzidos os instrumentais finos de trabalho (pinças, tesouras, porta-agulhas, aspirador, etc.). Todos os procedimentos, inclusive suturas e anastomoses, podem ser realizados. A retirada de órgãos se faz pelo umbigo ou pequenas inguinotomias, podendo também se fragmentar os órgãos ou tumores dentro de sacos plásticos introduzidos, removendo-se os fragmentos pela boca do saco exteriorizado no umbigo. A colectomia (para tratamento de aganglionose congênita ou tumores) tem sido realizada pela equipe somente por via transanal, utilizandose a laparoscopia somente para ligadura de vasos mesocólicos não alcançáveis pelo ânus.

Os orifícios dos trocartes eram fechados apenas no plano músculo-aponeurótico com um ponto de fio absorvível de poliglactina. A pele era fechada apenas com fitas adesivas durante 7 a 10 dias. Analgésicos eram prescritos por 1 a 3 dias, se necessário.

A dieta oral em casos sem suturas gastrintestinais era reiniciada de 1 a 8 horas de pós-operatório, dependendo do 
tipo da operação. Havendo boa aceitação, a maioria das crianças recebia alta hospitalar no mesmo dia ou no seguinte. Em casos de suturas gastrintestinais, a dieta era reiniciada após 24 ou 48 horas, caso se constatasse bom peristaltismo (ruídos intestinais e apetite presentes, eliminação de flatos ou evacuação).

As atividades físicas que apresentassem risco de quedas ou golpes eram limitadas nos primeiros 7 a 30 dias, dependendo da operação e se havia suturas intra-abdominais.

Todos os pacientes foram registrados em fichas de dados protocolados, arquivados no prontuário para seguimento evolutivo, incluindo identificação, quadro clínico, diagnóstico, patologia, detalhes da operação, pós-operatório e acompanhamento, que vem sendo realizado até a presente data. Para análise da estética abdominal, após 30 e 90 dias, os pais foram interrogados sobre a satisfação com as cicatrizes, registrando-se em escala de pontos que denominamos escala de satisfação estética (ESE): péssimo=1, regular $=2$, bom $=3$, muito bom $=4$, excelente $=5$.

Os resultados deste estudo preliminar prospectivo, ainda em andamento, foram baseados nos dados das fichas, prontuários e informações por colegas de outros estados ou cidades onde o autor EE realizou operações. Não foram incluídos pacientes submetidos à videocirurgia endourológica e as toracoscópicas.

\section{Resultados}

Houve uma grande variedade de afecções tratadas por via laparoscópica (Tabela 1). Considerando as mais freqüentes, as principais indicações para tratamento videolaparoscópico foram a doença do refluxo gastroesofágico (DRGE), abdome agudo inflamatório, apendicite, biópsias ou estadiamento de tumores, testículos não palpáveis, doenças ovarianas, obstrução por bridas, colecistectomia, aganglionose congênita, esplenectomias e trauma abdominal (indicada se paciente hemodinamicamente estável).

Não ocorreram complicações relacionadas à anestesia $\mathrm{e}$ ao pneumoperitônio. Não houve infecção hospitalar. Foi necessária transfusão de sangue em casos de trauma e no de uma criança com insuficiência renal crônica (IRC). Catorze pacientes vítimas de trauma e duas com IRC ou broncoespasmo necessitaram ou estavam em terapia intensiva $(2,7 \%)$. Houve 3 óbitos, conseqüentes à doença de base, por TCE em politrauma (2) e descompensação de IRC (1). Não ocorreram implantes tumorais nos orifícios dos trocartes. Catorze casos $(2,3 \%)$ necessitaram conversão para laparotomia pelo risco de hemorragia - trauma (8), esplenectomia para púrpura (1) e hemangioma hepático(1) - ou por intensas aderências peritoneais (2), ou por queima da fonte de luz ou da câmera durante a operação (2).

Complicações simples relacionadas à laparoscopia ocorreram em 6(1,0\%) crianças, como 2 com pequenos quelóides, alergia a iodo, enfisema subcutâneo e 2 pequeninas hérnias incisionais. Nos primeiros casos operados, quando não se fechava a camada muscular dos orifícios, ocorreu pequena hérnia incisional com epíploon em duas crianças (0,3\%). Desde 1996 não se constataram outros casos. Os detalhes técnicos específicos e as intercorrências secundárias a cada tipo de afecção fogem ao escopo deste trabalho.

Considerando somente a indicação mais comum nesta casuística, a DRGE, no seguimento de 1 a 5 anos houve duas recidivas do refluxo $(1,6 \%)$, sendo uma criança desnutrida com diarréia crônica, reoperada por via aberta em outro serviço, e outra neuropata que reapresentou refluxo após gastrostomia endoscópica, sendo reoperada por videolaparoscopia. Em 6 crianças com DRGE a videocirurgia foi reoperação de cirurgia aberta convencional ou laparoscópica prévia, 5 delas de outros serviços.

Em 16 crianças foram realizadas duas ou mais laparoscopias, indicadas para o segundo tempo de orquipexia (técnica de Fowler-Stephens), biópsias, lises repetidas de bridas, manipulação de cateteres de diálise e segunda avaliação em tumores ou trauma. Vinte e duas crianças $(3,7 \%)$ não voltaram para seguimento tardio, conforme a orientação ambulatorial.

Com o passar do tempo, as cicatrizes dos orifícios tendem a tornar-se planas e, em 94 crianças, desapareceram quase totalmente após 36 meses. $\mathrm{O}$ aspecto estético, analisado pela ESE nos últimos 368 pacientes, foi considerado excelente (ESE médio 4,6) por quase todos os pais e pelos médicos da equipe.

Houve rápida recuperação pós-operatória das crianças, notando-se boa movimentação com pouca dor e pouco uso de analgésicos, muito curto ou nenhum período de íleo pósoperatório a depender do procedimento.

\section{Discussão}

A videocirurgia em pediatria é um avanço recente, embora Gans e Berci já tivessem realizado laparoscopia com visão ocular direta ainda em $1971^{2}$. Naquela época, a laparoscopia era aplicada como método diagnóstico e para pequenas biópsias. Depois da primeira colecistectomia videolaparoscópica em $1987^{2}$, as técnicas por vídeo em adultos cresceram rapidamente e, nas crianças, esperaram apenas a miniaturização do equipamento.

Entre 1991 e 1993 foram relatados os primeiros resultados promissores em refluxo gastroesofágico, criptorquia, apendicite aguda e outras afecções $1,3,6$. Desde então a videolaparoscopia tornou-se importante na redução da morbidade perioperatória, demonstrando inúmeras vantagens em crianças, comparadas às operações abertas, inclusive com menor custo financeiro, ao contrário do que muitos imaginam ${ }^{1,4,5}$.

A realização da videocirurgia requer treinamento especial do cirurgião devido à visão bidimensional em um monitor de vídeo ou televisão, bem diferente da visão tridimensional bi-ocular habitual em cirurgia aberta. Além 
disso, o trabalho com instrumentos sob visão bidimensional é mais difícil em crianças pequenas devido ao menor espaço abdominal, se comparado ao dos adultos ${ }^{2}$. Outro fator novo e importante em laparoscopia é o pneumoperitônio por dióxido de carbono, o qual requer do anestesista a monitorização contínua do $\mathrm{CO}_{2}$ expirado com capnógrafo, controlando a ventilação para manter um nível máximo de 35-40 $\mathrm{mmHg}^{7}$. Como demonstrado em nossa casuística, em que não se observou nenhum efeito de hipercapnia, o pneumoperitônio é bem tolerado por crianças, quando não se pratica níveis de pressão intra-abdominal superiores a 10 mmHg.

As principais aplicações nas quais a laparoscopia é considerada verdadeiro avanço terapêutico por nós e outros cirurgiões são a DRGE com ou sem hérnia hiatal ${ }^{8,9}$, a criptorquia alta ${ }^{10,11}$, o abdome agudo com dúvida diagnóstica, sobretudo em meninas adolescentes ${ }^{1,12,13}$, o trauma abdominal fechado ou aberto (faca ou arma de fogo) ${ }^{14}$, a obstrução por bridas ${ }^{15,16}$, o megacólon congênito (dispensando a colostomia) $)^{6,17}$, as doenças do ovário ${ }^{18,19}$, a colecistectomia $^{20}$, a esplenectomia ${ }^{6,21}$, o diagnóstico por biópsias $1,6,22$, o estadiamento de doença de Hodgkin ou outros tumores ${ }^{23,24}$, as hérnias diafragmáticas, o volvo gástrico ${ }^{25}$, os cistos ou divertículos, a investigação de icterícia e hérnia inguinal contralateral ${ }^{1,26,27}$. Além das indicações cirúrgicas citadas na Tabela 1 , há muitas outras já publicadas que ainda não utilizamos.

O tratamento padrão laparoscópico na gastroenterologia em adultos é a colecistectomia. Em pediatria, a litíase biliar é menos freqüente, mas a videolaparoscopia é o melhor acesso cirúrgico ${ }^{1,6,28}$. Em crianças, consideramos que, atualmente, o tratamento laparoscópico padrão é o da DRGE, considerando a grande incidência e o potencial de morbidade da doença ${ }^{4,8}$. Entre as diversas técnicas utilizadas, a principal é a fundoplicatura a Nissen, envolvendo todo o contorno do esôfago abdominal em 360 graus. Esta técnica apresenta os menores riscos de recidiva, sendo especialmente indicada em crianças neuropatas e desnutridas ${ }^{1,8}$. Outras técnicas utilizadas são de Boix-Ochoa e Thal, onde o esôfago é envolvido em menos de 180 graus, associado assim a menos disfagia pós-operatória, mas a um risco maior de recidiva ${ }^{9,29}$. Em nossa casuística, obtivemos um índice de recidiva $(2,7 \%)$ inferior à média na literatura (5-20\%), apesar do período de acompanhamento de até cerca de 5 anos $^{4,8,9,29}$. Vários autores têm demonstrado menor índice de complicações perioperatórias da laparoscopia em comparação com as operações abertas, com mesmas taxas de cura da doença do refluxo gastroesofági$\mathrm{co}^{4,8,29}$.

Em outros serviços com grandes casuísticas, a apendicectomia laparoscópica é a operação mais realizada ${ }^{12,13}$, sendo indicada especialmente em casos de crianças obesas ou acima de 10 anos, quadros atípicos, meninas adolescentes ou apendicite complicada. Consideramos que, em crianças magras e sem evidência de plastrão, a operação convencional com pequena incisão ainda é mais vantajosa.
Na cirurgia oncológica pediátrica, a introdução da videolaparoscopia para a realização de procedimentos, como biópsias e estadiamentos, com mínima invasão e rápida recuperação pós-operatória, tem permitido o início precoce de quimioterapia para os diversos tumores, com menor risco de deiscências de parede e infecção ${ }^{22,23}$. Em operações em cavidade aberta, normalmente se recomenda iniciar a quimioterapia ou radioterapia após a cicatrização da laparotomia (8 a 10 dias), mesmo assim com alto risco de deiscência, infecção e hérnias incisionais. Após a laparoscopia, a quimioterapia pode ser iniciada em 24 a 48 horas. A própria redução da dor e hemorragia operatórias pela laparoscopia são fatores positivos na qualidade de vida das crianças com câncer.

A principal indicação para laparoscopia em grandes centros europeus é a lise de bridas em casos de obstrução intestinal ${ }^{1}$, e consideramos a melhor opção quando não haja antecedente de peritonite difusa ou esclerose peritoneal por diálises. A indicação da operação deve ser precoce ${ }^{16}$. Devido à distensão das alças, a introdução dos trocartes deve ser efetuada sob visão direta do peritônio, pois o principal motivo de complicação e conversão para laparotomia é a perfuração intestinal, além da intervenção tardia, a qual freqüentemente evidencia necrose intestinal ${ }^{15,16}$.

O trauma pediátrico é uma das principais causas de morbi-mortalidade em crianças. A tendência atual é o tratamento conservador no trauma fechado com lesão de órgãos sólidos, permanecendo a criança internada muitas vezes por tempo prolongado, monitorando-se a evolução das lesões por exames de imagem e estado hemodinâmico. O hemoperitôneo geralmente acarreta dor e íleo paralítico prolongado. A perfuração de víscera oca pode passar desapercebida por dias, sobretudo na criança comatosa ${ }^{30}$. A laparoscopia permite avaliação precoce da cavidade, lavagem do pneumoperitôneo, cauterizações e rafias, com rápida recuperação, menor chance de infecção hospitalar; permite também evitar, por exemplo, uma ruptura do baço em dois tempos ${ }^{14}$.

No trauma por arma branca ou arma de fogo, a conduta usual é a laparotomia imediata. A freqüência de laparotomias em que não se encontram lesões intra-abdominais (laparotomias brancas ou desnecessárias) varia de 4 a $52 \%^{30}$. Estes índices seriam minimizados com a realização de um melhor exame e acompanhamento clínico, além de uma laparoscopia precoce. A nossa conduta é a avaliação da cavidade por laparoscopia quando disponível, podendo-se inclusive utilizar o orifício penetrante para introdução de uma ótica. Lavagem da cavidade, debridamento, rafias e drenagem podem ser facilmente realizadas sem laparotomia, sendo a recuperação muito mais rápida e com melhor estética do que com laparotomia. Bons exames de imagem e exame clínico reduzem bastante o índice de laparotomias ou laparoscopias desnecessárias ${ }^{1,30}$. Nos casos de instabilidade hemodinâmica e ruptura diafragmática, a videocirurgia e o pneumoperitôneo artificial são contra-indicados. 
Outras aplicações da laparoscopia incluem operações urológicas como a nefrectomia ${ }^{31}$, a adrenalectomia ${ }^{32}$, o refluxo vésico-ureteral ${ }^{33}$, a varicocele ${ }^{1}$, a pieloplastia ${ }^{1}$, etc. Entretanto, as vantagens não foram ainda estabelecidas, pois, nas operações convencionais, cirurgiões habilidosos utilizam pequenas incisões posteriores ou suprapúbicas esteticamente aceitáveis, manipulando-se o retroperitônio sem íleo paralítico ${ }^{6}$.

Indicações menos freqüentes, mas reconhecidamente vantajosas, por videolaparoscopia, em que obtivemos ótimo resultado, são para o tratamento de invaginação intestinal sem ressecção ${ }^{34}$, cistos omentais ou mesentéricos ${ }^{1}$, enterectomia para divertículo de Meckel ${ }^{1,6}$, duplicidade e linfangioma por via trans-umbilical, esplenectomia com ou sem colecistectomia ${ }^{6,21}$, má rotação intestinal ${ }^{35}$, volvo gástrico $^{35,36}$, baço errante ${ }^{1,}$, hérnias diafragmáticas como a hiatal e de Morgagni, confecção de pertuito de cateterismo cecal para enemas (cirurgia de Malone) ${ }^{37}$, manipulação de cateteres de diálise ou derivação liquórica, debridamento em pancreatite aguda e muitas outras aplicações.

Ainda é bastante controversa a indicação da videolaparoscopia para tratamento da estenose hipertrófica do piloro ${ }^{6,38}$, hérnias inguinais na criança ${ }^{26,27}$, fase inicial de apendicite em crianças pequenas ou magras ${ }^{1,12,13}$, biópsia renal e varicocele ${ }^{32}$. Em todos estes casos, as operações por via aberta por mini-incisões estéticas são fáceis e de baixa morbidade. Admite-se, porém, que, com os novos instrumentos super finos, de 2 ou $3 \mathrm{~mm}$ de diâmetro, pode haver maior vantagem em aplicar a videolaparoscopia nestes casos. Consideramos aplicável a videolaparoscopia em varicocele e pesquisa de hérnia contralateral somente em crianças grandes. A maioria absoluta das biópsias renais pode ser efetuada por punção.

Esta nova via de acesso permite realizar as mesmas operações efetuadas por via aberta, porém evita a secção dos planos da parede abdominal e a exposição das vísceras $^{1,2}$. Tem sido demonstrado que, devido ao menor trauma, há uma menor resposta inflamatória e menos alterações endócrinometabólicas ou cardiovasculares ${ }^{1,28,39}$. Uma grande vantagem é a menor dor pós-operatória, associada a menor limitação da respiração, menor uso de analgésicos ${ }^{5,7}$ e rápido retorno às atividades da criança, que normalmente não colabora com o repouso.

Há menor ou mínimo sangramento, os orifícios deixam cicatrizes mínimas quando não desaparecem, obtendo-se o melhor efeito estético até agora possível. Quando se fecha o plano músculo-aponeurótico no orifício, não ocorrem hérnias incisionais. As micro-incisões apresentam menor risco de aderências à parede, serosidade e hematomas. Utilizam-se muito menos fios de sutura, pois não há laparotomia. Pela não exposição e menor manipulação visceral, quase não ocorre íleo paralítico e distensão intestinal pósoperatória, permitindo a realimentação precoce da criança. O tempo necessário de internação torna-se menor, com menor risco de infecção hospitalar ${ }^{1,2,5}$.
Comparados com as operações abertas, a perda de líquido e o distúrbio hidroeletrolítico pela evaporação nas serosas são desprezíveis. A microcâmera permite ver toda a cavidade, as estruturas com grande aumento e detalhes, impossível com laparotomias não extensas, ocorrendo também menor risco de se esquecer gazes ou outros objetos na cavidade.

As principais desvantagens relatadas em relação à videolaparoscopia são a necessidade de um treinamento especial em videocirurgia, o alto custo dos equipamentos e um risco maior de alguns tipos de lesões como perfuração de vísceras por trocartes, cauterização de vias biliares, dificuldade de controle de grandes hemorragias $1,2,40$. Acidose e hipercapnia podem ocorrer se a ventilação não for adequadamente efetuada com níveis seguros de pressão intraabdominal de $\mathrm{CO}_{2}{ }^{1,7}$. Com seleção adequada dos pacientes e realizando técnica cuidadosa, não nos ocorreu nenhuma dessas complicações.

O custo dos equipamentos de videocirurgia é alto, com tendência a queda devido à concorrência entre os fabricantes. Entretanto, como os hospitais cobram das empresas de saúde uma taxa pelo uso do equipamento, o custo é recuperado em um ou dois anos. O uso de material reutilizável e a redução do tempo operatório colaboram na redução do custo $^{5}$. Considerando vários fatores como material consumido, honorários médicos, medicamentos, prejuízo em dias escolares pela internação, curativos e outros cuidados, autores americanos compararam os custos e relataram que há um menor custo geral (hospitalar, familiar e social) do que com operações semelhantes por laparotomia ${ }^{4,5}$.

Concluímos que a videolaparoscopia parece ser um grande avanço da cirurgia pediátrica moderna, permitindo tratar de modo menos invasivo e com muita segurança grande parte das afecções abdominais em crianças de todas idades, demonstrando-se inúmeras vantagens sobre as laparotomias, quando bem indicadas e realizadas dentro dos princípios cirúrgicos e anestésicos corretos.

\section{Referências bibliográficas}

1. Moir CR. Gastrointestinal endoscopy, laparoscopy and other noninvasive surgical techniques. In: O'Neill Jr JA, Rowe M, Grosfeld JL, Fonkalsrud EW, Coran AG, eds. Pediatric Surgery. $5^{\mathrm{a}}$ ed. St Louis: Mosby-Year Book, Inc; 1998. p.1233-51.

2. Lobe TE. Basic laparoscopy. In: Lobe TE, Schropp KP, eds. Pediatric laparoscopy and thoracoscopy. $1^{\mathrm{a}}$ ed. Philadelphia: WB Saunders Co;1994. p.81-92.

3. Georgeson KE. Laparoscopic gastrostomy and fundoplication. Pediatr Ann 1993; 22:675.

4. Coster DD, Bower WH, Wilson VT, Butler DA, Locker SC, Brebrick RT. Laparoscopic Nissen fundoplication: a curative, safe and cost-effective procedure for complicated gastroesophageal reflux disease. Surg Laparosc Endosc 1995; 5:111-7. 
5. Luks FI; Logan J, Breuer CK, Kurkchubasche AG, Wesselhoeft Jr CW, Tracy Jr TF. Cost-effectiveness of laparoscopy in children. Arch Pediatr Adolesc Med 1999; 153:965-8.

6. Heloury Y, Leclair MD, Plattner V. La place de la coelioscopie en chirurgie pediatrique. Ann Chir 1998; 52:1003-7.

7. Takrouri MS. Anesthesia for laparoscopic general surgery: a special review. Middle East J Anesthesiol 1999; 15:39-62.

8. Rothenberg SS. Experience with 220 consecutive laparoscopic Nissen fundoplications in infants and children. J Pediatr Surg 1998; 33:274-8.

9. Esteves E, Neto MO, Lima FLS, Penna HM, Pereira RE. Operação de Boix-Ochoa videolaparoscópica. Anais do III Congresso de Cirurgia Pediátrica do Cone Sul da América; 1998 Set 27-30; Viña del Mar (Chile). Resumo V-15.

10. Lanna JCBD, Sobrinho JMDL. Distopias testiculares. In: Maksoud JG, ed. Cirurgia Pediátrica. $1^{\mathrm{a}}$ ed. Rio de Janeiro: Revinter; 1998. p. 689-705.

11. Khatwa UA, Menon PS. Management of undescended testis. Indian J Pediatr 2000; 67:449-54.

12. Lejus C, Delile L, Plattner V, Baron M, Guillou S, Heloury Y, et al. Randomized, single blinded trial of laparoscopic versus open appendectomy in children. Anesthesiology 1996; 84:801-6.

13. El Ghoneimi A, Valla JS, Limonne B, Valla V, Montupet $P$, Chavrier Y, et al. Laparoscopic appendicectomy in children: report of 1379 cases. J Pediatr Surg 1994; 29:786-9.

14. Gandhi RR, Stringel G. Laparoscopy in pediatric abdominal trauma. J Soc Laparoendosc Surg 1997; 1:349-51.

15. Van der Zee DC, Bax NM. Management of adhesive bowel obstruction in children is changed by laparoscopy. Surg Endosc 1999; 13:925-7.

16. Becmeur F, Besson R. Treatment of small-bowel obstruction by laparoscopy in children multicentric study. Eur J Pediatr Surg 1998; 8:343-6.

17. Wulkan ML, Georgeson KE. Primary laparoscopic endorectal pull-through for Hirschsprung's disease in infants and children. Semin Laparosc Surg 1998; 5:9-13.

18. Jawad AJ, Al-Meshari A. Laparoscopy for ovarian pathology in infancy and childhood. Pediatr Surg Int 1998; 14:62-5.

19. Moir CR. Laparoscopy for the female patient. In: Holcomb GW III, ed. Pediatric endoscopic surgery. $1^{\text {a }}$ ed. Norwalk (Connecticut): Appleton \& Lange; 1994. p.101.

20. Holcomb GW III, Sharp KW, Neblett WW III, Morgan WM III, Pietsch JB. Laparoscopic cholecystectomy in infants and children: modifications and cost analysis. J Pediatr Surg 1994; 29:900-4.

21. Curran TJ, Foley MI, Swanstrom LL, Campbell TJ. Laparoscopy improves outcomes for pediatric splenectomy. J Pediatr Surg 1998; 33:1498-500.

22. Holcomb GW III, Tomita SS, Haase GM, Dillon PW, Newman $\mathrm{KD}$, Applebaum $\mathrm{H}$, et al. Minimally invasive surgery in children with cancer. Cancer 1995; 76:121-8.

23. Holcomb GW III. Minimally invasive surgery for solid tumors. Semin Surg Oncol 1999; 16:184-92.

24. Baccarani U, Carroll BJ, Hiatt JR, Donini A, Terrosu G, Decker $\mathrm{R}$, et al. Comparison of laparoscopic and open staging in Hodgkin disease. Arch Surg 1998; 133:517-22.

25. Odaka A, Shimomura K, Fujioka M, Inokuma S, Takada S, Yamada H, et al. Laparoscopic gastropexy for acute gastric volvulus: a case report. J Pediatr Surg 1999; 34:477-8.

26. Montupet P, Esposito C. Laparoscopic treatment of congenital inguinal hernia in children. J Pediatr Surg 1999; 34:420-3.
27. Van Glabeke E, Khairouni A, Gall O, Le Pointe HD, Jaby O, Larroquet $\mathrm{M}$ et al. Laparoscopic diagnosis of contralateral patent processus vaginalis in children under 1 year of age with unilateral inguinal hernia: comparison with herniography. J Pediatr Surg 1999; 34:1213-5.

28. Jakeways MS, Mitchell V, Hashim IA, Chadwick SJ, Shenkin A, Green CJ, et al. Metabolic and inflammatory responses after open or laparoscopic cholecystectomy. Br J Surg 1994; 81:127-31.

29. Van der Zee DC, Arends NJ, Bax NM. The value of 24-h pH study in evaluating the results of laparoscopic antireflux surgery in children. Surg Endosc 1999; 13:918-21.

30. Eichelberger MR, Moront M. Abdominal trauma. In: O'Neill Jr JA, Rowe MI, Grosfeld JL, Fonkalsrud EW, Coran AG, eds. Pediatric Surgery. $5^{\text {a }}$ ed. St Louis: Mosby-Year Book, Inc; 1998. p.261-84.

31. Davies BW, Najmaldin AS. Transperitoneal laparoscopic nephrectomy in children. J Endourol 1998; 12:437-40.

32. Nyirady P, Merksz M, Pirot L, Altorjay A, Pelenyi A, Kiss A, et al. Laparoszkopos beavatkozasok a gyermekurologiaban. Orv Hetil 1999; 140:1403-6.

33. Homsy YL. Current thoughts on the place of laparoscopy in pediatric urology. Urology 1994; 44:815.

34. Schier F. Experience with laparoscopy in the treatment of intussusception. J Pediatr Surg 1997; 32:1713-4.

35. Bass KD, Rothenberg SS, Chang, JHD. Laparoscopic Ladds's procedure in infants with malrotation. J Pediatr Surg 1998; 33:279-81.

36. Esteves E, Ottaiano Neto M, Salgado CM, Pereira RE. Gastropexia laparoscópica para volvo gástrico. Anais do III Congresso de Cirurgia Pediátrica do Cone Sul da América; 1998 Set 27-30; Viña del Mar (Chile). Resumo V-24.

37. Van Savage JG, Yohannes P. Laparoscopic antegrade continence enema in situ appendix procedure for refractory constipation and overflow fecal incontinence in children with spina bifida. J Urol 2000; 164:1084-7.

38. Fujimoto T, Lane GJ, Segawa O, Esaki S, Miyano T. Laparoscopic extramucosal pyloromyotomy versus open pyloromyotomy for infantile hypertrophic pyloric stenosis: which is better? J Pediatr Surg 1999; 34:370-2.

39. Gentili A, Iannettone CM, Pigna A, Landuzzi V, Lima M, Baroncini S. Cardiocirculatory changes during videolaparoscopy in children: an echocardiographic study. Paediatr Anaesth 2000; 10:399-406.

40. Chen MK, Schropp KP, Lobe TE. Complications of minimalaccess surgery in children. J Pediatr Surg 1996; 31:1161-5.

\section{Endereço para correspondência:}

Dr. Edward Esteves

Rua T-65, $\mathrm{n}^{\circ} 1.077$, ap. 1.303-B, Setor Bueno

CEP 74230-120 - Goiânia, GO

Fones: (62) 941.6009, 215-3859 - Fax (62) 281.5508

E-mail: edward@doctor.com 